\title{
Characterization and closed-loop AO performance of a liquid deformable mirror
}

\author{
Eric S. ten Have ${ }^{a}$ and Gleb Vdovin ${ }^{a, b}$ \\ ${ }^{a}$ Delft University of Technology, Electronic Instrumentation Laboratory, \\ Mekelweg 4, 2628 CD Delft, the Netherlands; \\ ${ }^{b}$ Flexible Optical B.V., Polakweg 10-11, 2288 GG Rijswijk, the Netherlands
}

\begin{abstract}
Closed-loop adaptive optical correction using a deformable mirror based on the total internal reflection (TIR) from an electrostatically deformed liquid-air interface was performed on a collimated beam of a HeNe-laser that was aberrated by a rotating phase disk. The frequency response of the system was measured and the influence of liquid surface motion in absence of external optical aberrations on the liquid mirror was characterized. The performance of the $\mathrm{AO}$ system was determined for static and dynamic aberrations for various sets of system parameters.
\end{abstract}

Keywords: adaptive optics, closed-loop operation, deformable mirror, total internal reflection

\section{INTRODUCTION}

Researchers have attempted to exploit liquid-air-interfaces as deformable mirror surfaces in a variety of applications, from parabolic mirrors that used rotating mercury ${ }^{1-3}$ to magnetically deformed liquid mirrors that used an additional reflection layer on top of the magnetic liquid. ${ }^{4}$ In all these applications the light was reflected from the air side of the liquid-air interface which means that the deformation of the liquid had to be induced from within the liquid. The method described here uses total internal reflection (TIR) in which the light is coupled into the mirror by a coupling prism to propagate through the liquid so that it is reflected by the liquid side of the liquid-air interface (Fig. 1). In this case the electrode structure is placed above the liquid and is separated from the interface by an air gap. This non-dissipative method has been used for a novel deformable mirror that uses electrostatical deformation of the liquid-air interface to shape the wavefront of the reflected light. In a previous publication the differential equation governing the static behavior of the mirror was numerically solved and stable closed-loop operation of the device was demonstrated for the first time. ${ }^{5}$ Here, we present the results of further work on the characterization of the liquid mirror in a closed-loop adaptive optical system.
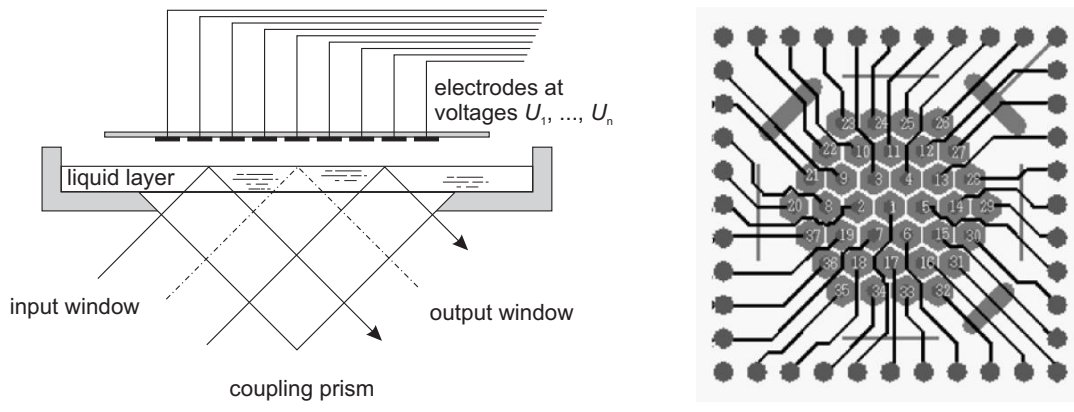

Figure 1. Setup of the liquid mirror (left) and geometry of the electrode structure (right).

Further author information: (Send correspondence to E.S. ten Have)

E.S. ten Have: E-mail: e.s.tenhave@tudelft.nl

G. Vdovin: E-mail: gleb@okotech.com, http://www.okotech.com, Telephone: +31 (0)70 262 94-20

Unconventional Imaging, Wavefront Sensing, and Adaptive Coded Aperture Imaging and Non-Imaging Sensor Systems, edited by Jean J. Dolne, Thomas J. Karr, Victor L. Gamiz, Stanley Rogers, David P. Casasent, Proc. of SPIE Vol. 8165, 816504 - (C) 2011 SPIE · CCC code: 0277-786X/11/\$18 · doi: 10.1117/12.893758 

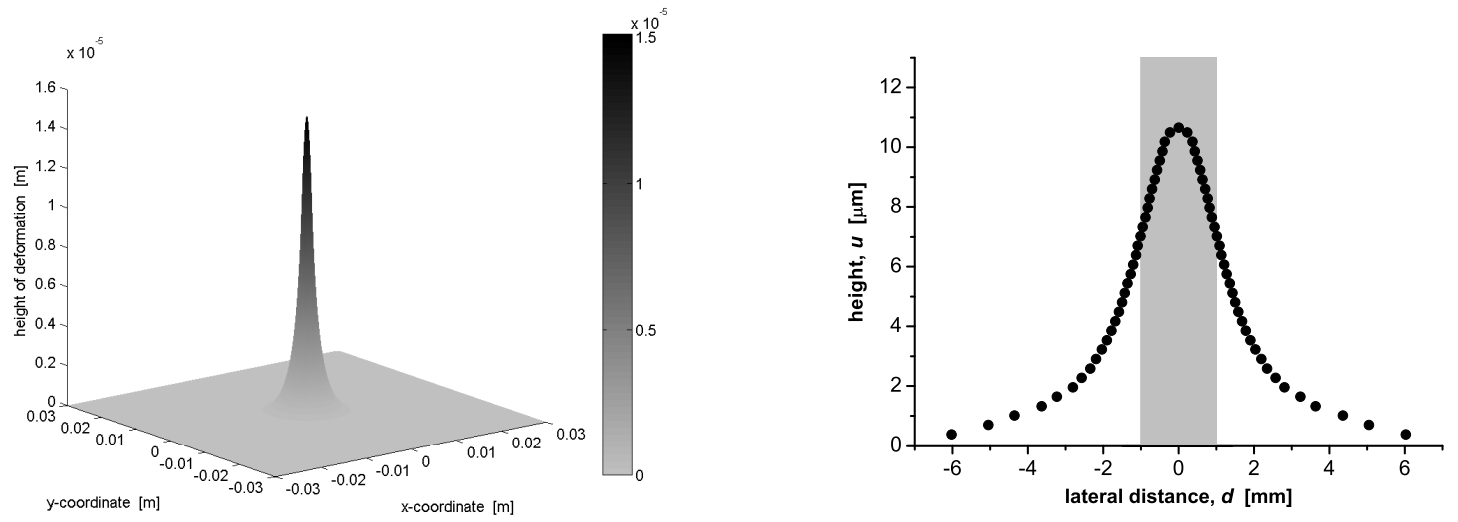

Figure 2. Shape of the electrostatically deformed liquid surface obtained through solving Eq. 2 numerically (left) and by measurement with an interferometer (right), the lateral size of the actuator is indicated by the shaded area.

\section{THEORETICAL BACKGROUND}

In case of the electrostatically deformed liquid three forces act on each element of the liquid surface, viz. the electrostatic force, surface tension, and gravity. The equilibrium situation in which all forces cancel each other is described by:

$$
F_{\text {elec }}-F_{\text {grav }}-F_{\text {surface }}=0 .
$$

The shape, $u$, of the deformation caused by an electrode positioned above the liquid surface is described by the solution of a differential equation: ${ }^{5}$

$$
\frac{\varepsilon \varepsilon_{0} U^{2}(x, y)}{2 d^{2}}-(\rho g-k) u-T\left(\frac{\partial^{2} u}{\partial x^{2}}+\frac{\partial^{2} u}{\partial y^{2}}\right)=0
$$

with $\varepsilon$ the dielectric constant of the gas filling the gap, $U$ the electric potential, $d$ the distance between the electrode and the liquid surface, $\rho$ the density of the liquid, $g$ the gravitational constant, $k$ the electric constant ( $k>0$ for gels and $k=0$ for liquids), and $T$ the surface tension of the liquid. The differential equation is valid for a nonzero conductivity of the liquid and considers the geometry of the electrode through the electric potential, $U(x, y)$, and the resulting electric field. Fig. 2 (left) shows the shape of the liquid surface obtained through solving the differential equation numerically.

\section{MEASUREMENTS AND RESULTS}

The setup (Fig. 3) for the measurements included an interferometer (shown on the right) for the measurements of the surface deformation. The measured surface deformation of a single actuator is shown in Fig. 2 on the right.

\subsection{Open-loop characterization of the liquid mirror response}

During the experiment an aqueous solution of $80 \%$ glycerin was used as liquid and only a single actuator with a radius of about $r=0.1 \mathrm{~mm}$ was controlled by a signal generator. The voltage applied was $U=400 \mathrm{~V}$ and the distance between the actuator and the liquid surface was about $d=700 \mu \mathrm{m}$.

Instead of a wavefront sensor as in Fig. 3 the light reflected from the liquid mirror was focussed on a knife-edge and subsequently detected by a fast photodiode. The signals from the photodiode and the signal generator were then recorded with an oscilloscope. The open-loop frequency response of the mirror is shown in Fig. 4 in form of a Bode plot. The measured response was normalized to obtain a unity DC system gain. The high frequency asymptote has a slope of about $-20 \mathrm{~dB} /$ decade and suggests a first order system while the peak at a frequency 


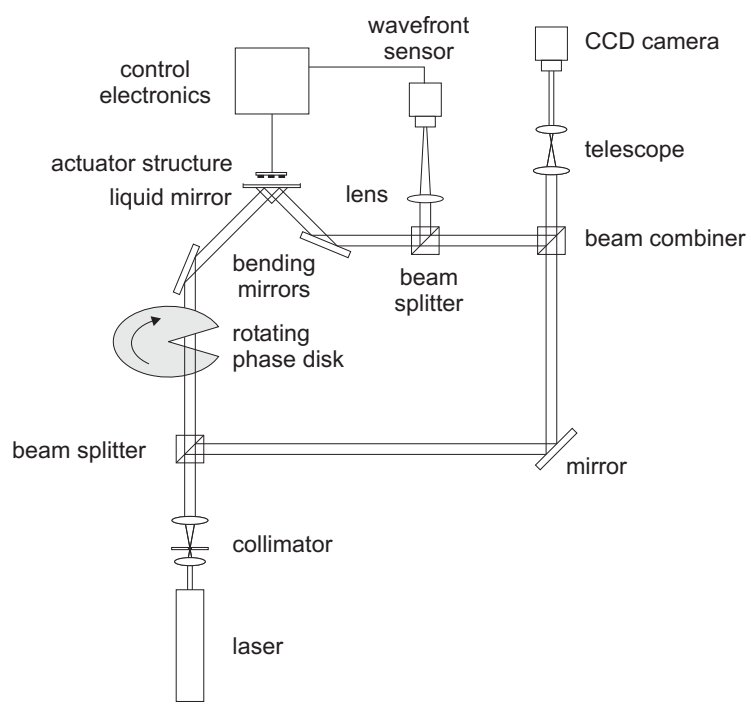

Figure 3. Setup for the closed loop measurements including an interferometric arm for the measurement of the surface deformation.

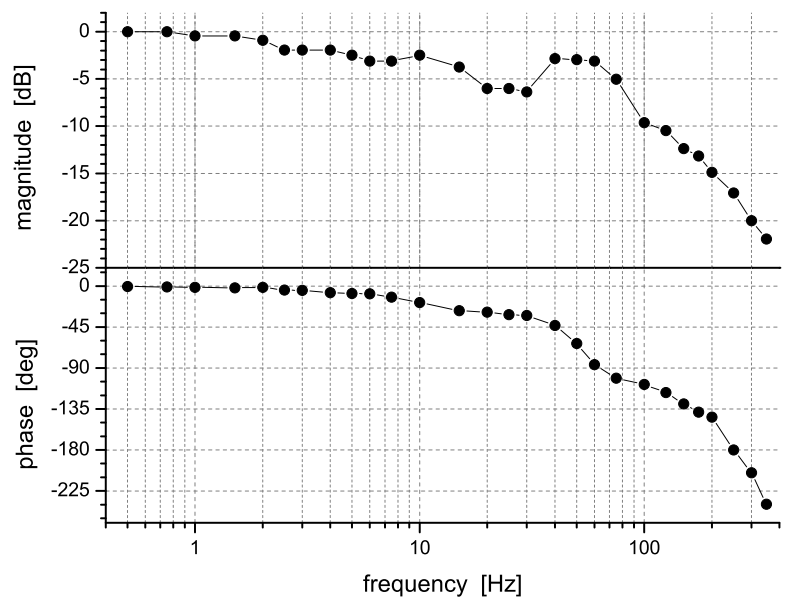

Figure 4. Frequency response of the liquid mirror device.

of $f_{n}=50 \mathrm{~Hz}$ indicates at least a system of second order or greater. The gain crossover frequency and the phase crossover frequency of the system are given by $f_{g c}=1 \mathrm{~Hz}$ and $f_{p c}=250 \mathrm{~Hz}$, respectively, obtaining a gain margin of $G M=17 \mathrm{~dB}$ and a phase margin of $P M=179^{\circ}$, thus fulfilling the stability criteria, $f_{g c}<f_{p c}$ and $G M<0$ at $f_{p c}$.

\subsection{Performance of the closed-loop AO system}

The closed-loop experiments were performed on an aqueous solution of $80 \%$ glycerin with 37 actuators of $1.9 \mathrm{~mm}$ diameter arranged in a hexagonal geometry (Fig. 1 right) with an air gap of $<1 \mathrm{~mm}$ resulting in a maximum stroke of about $18 \mu \mathrm{m}$. The effective pupil size was $9 \mathrm{~mm}$ and the maximum feedback frequency was $36 \mathrm{~Hz}$ while the wavefront sensor consisted of 127 subapertures arranged in a hexagonal geometry.

Initially, the static correction performance of the liquid mirror was measured: different locations of the phase disk were used to aberrate the wavefront of the laser beam in a first step without feedback, while the liquid 

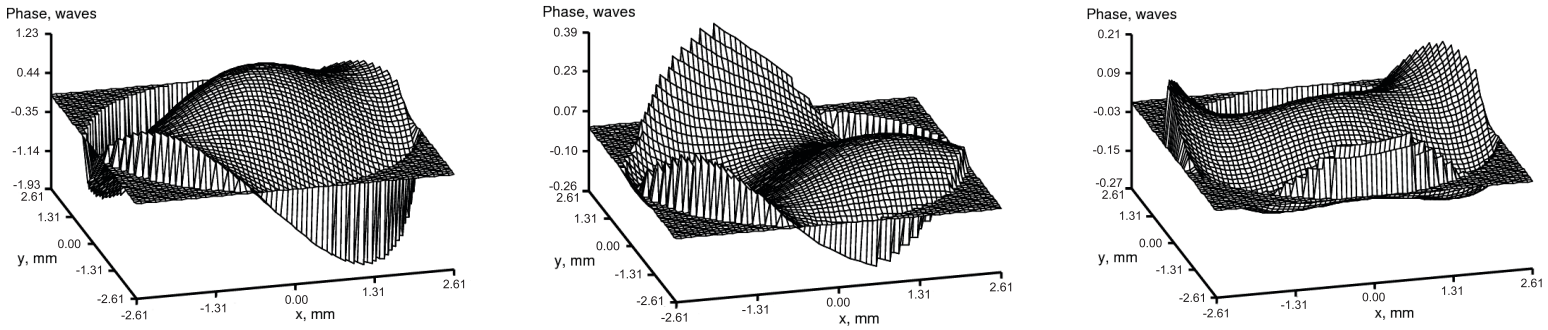

Figure 5. Wavefronts of an aberrated (left), corrected (middle), and undisturbed beam (right).
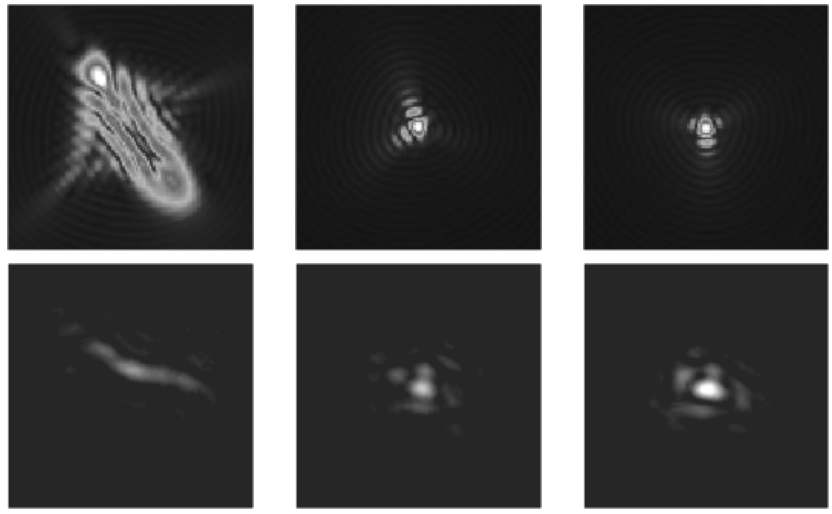

Figure 6. Far field images of the beam - top row: reconstruction from the wavefront sensor data, bottom row: measurement with the CCD camera behind a microscope objective. The images show the aberrated (left), corrected (middle), and undisturbed beams (right).

mirror was in its initial flat position, and in a second step with the feedback engaged, when the wavefronts were corrected. From the recorded wavefronts the correction improvement was calculated as the ratio of residual wavefront rms-errors and Strehl numbers.

Figs. 5 and 6 show examples of results of the static wavefront correction: the images show the wavefronts (Fig. 5), the simulated far field (Fig. 6 top row), and the measured far field (Fig. 6 bottom row) for an aberrated (left), corrected (middle), and undisturbed beam (right). The amount of residual error and Strehl number of the beams shown is summarized in Tab. 1. On average the improvement, $I$, for all different positions of the phase disk was $I_{\mathrm{rms}}=6.44$ and $I_{\text {Strehl }}=438$ for the ratio of the measured rms errors and Strehl numbers, respectively.

Subsequently, the dynamic correction performance for different sets of parameters of the liquid mirror was measured with the rotating phase disk. The phase disk was built from a clear plastic disk treated with hair spray and had a wedge cut out to let the beam pass undisturbed. During the rotation of the phase disk the residual wavefront error with the feedback switched on and off was recorded (Fig. 7). Switching the feedback on or off during the passage of the cut out part of the disk assured that the static aberrations of the setup were

Table 1. Residual errors and Strehl numbers of the aberrated, corrected, and undisturbed beam.

\begin{tabular}{|cc|c|c|c|}
\hline & $\begin{array}{c}\text { aberrated } \\
\text { beam }\end{array}$ & $\begin{array}{c}\text { corrected } \\
\text { beam }\end{array}$ & $\begin{array}{c}\text { undisturbed } \\
\text { beam }\end{array}$ \\
\hline peak-to-valley & {$[\mu m]$} & 1.989 & 0.413 & 0.297 \\
rms & {$[\mu m]$} & 0.430 & 0.057 & 0.042 \\
Strehl & & 0.004 & 0.658 & 0.794 \\
\hline
\end{tabular}




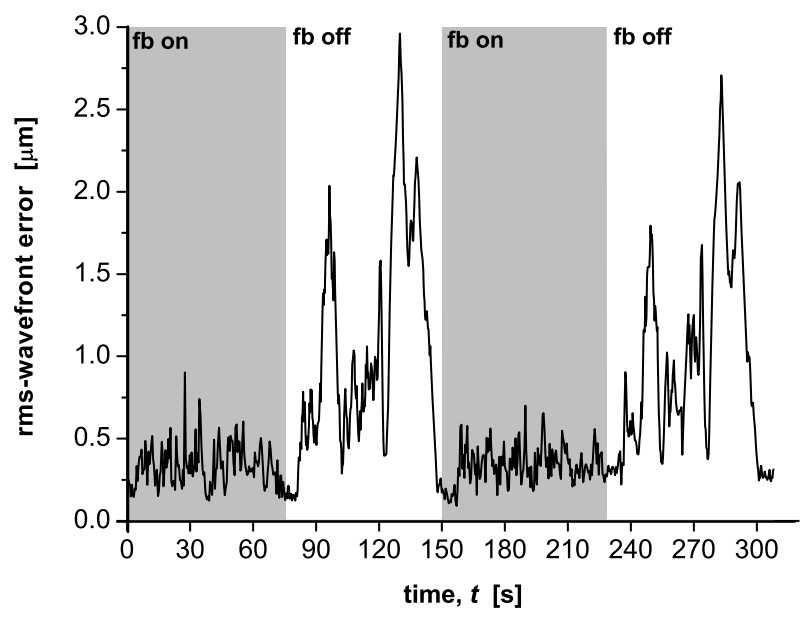

Figure 7. Measurement of the wavefront rms-error with the rotating phase disk, the passages with the activated feedback are shown with the grey background color. The feedback was switched on and off during the passage of the cut out part of the disk when the rms-error was especially low.
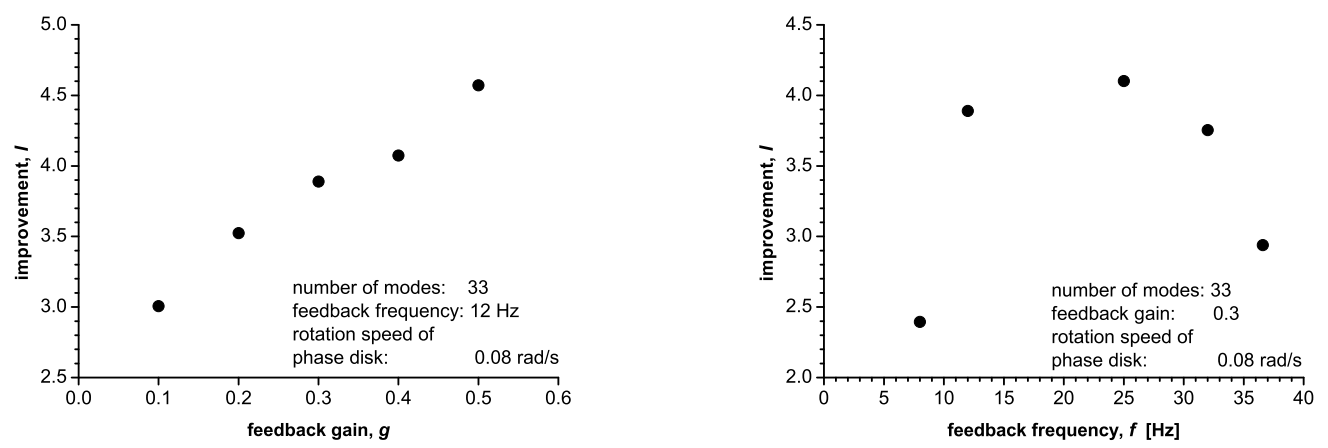

Figure 8. Dynamic correction performance of the liquid mirror setup in terms of improvement, the ratio of the measured wavefront error induced by the rotating phase disk with and without closing the feedback loop of the liquid mirror system, as a function of feedback gain (left) and feedback frequency (right).

corrected for even while the feedback was switched off. The residual rms-error of the wavefront was averaged for multiple rotations of the disk to compute the improvement factor by dividing the average rms-error without feedback by the average rms-error with active feedback. Without feedback the conditions remained stable for all measurements, so that the rms-error was relatively constant for all measurements only depending on the rotation speed of the disk, while the performance of the liquid mirror system with active feedback depended strongly on the chosen feedback parameters (Fig. 8).

The error signal from the measured wavefronts can be deconstructed into a superposition of Zernike modes. An example of a more detailed analysis of the first two Zernike modes (tip and tilt) is shown in Fig. 9: the deflection of the beam calculated from the tip and tilt mode coefficients is shown in the polar graphs. The bar graphs show histograms of the amplitude and angle of the deflection. Fig. 10 shows the beam deflection by the movement of the liquid only excited by ambient vibrations. The polar distribution plots (Fig. 10 top left without feedback and top right with feedback) show the existence of a preferred direction along which the laser beam is deflected. The bar graphs show histograms of the amplitude and angle of the deflection. 

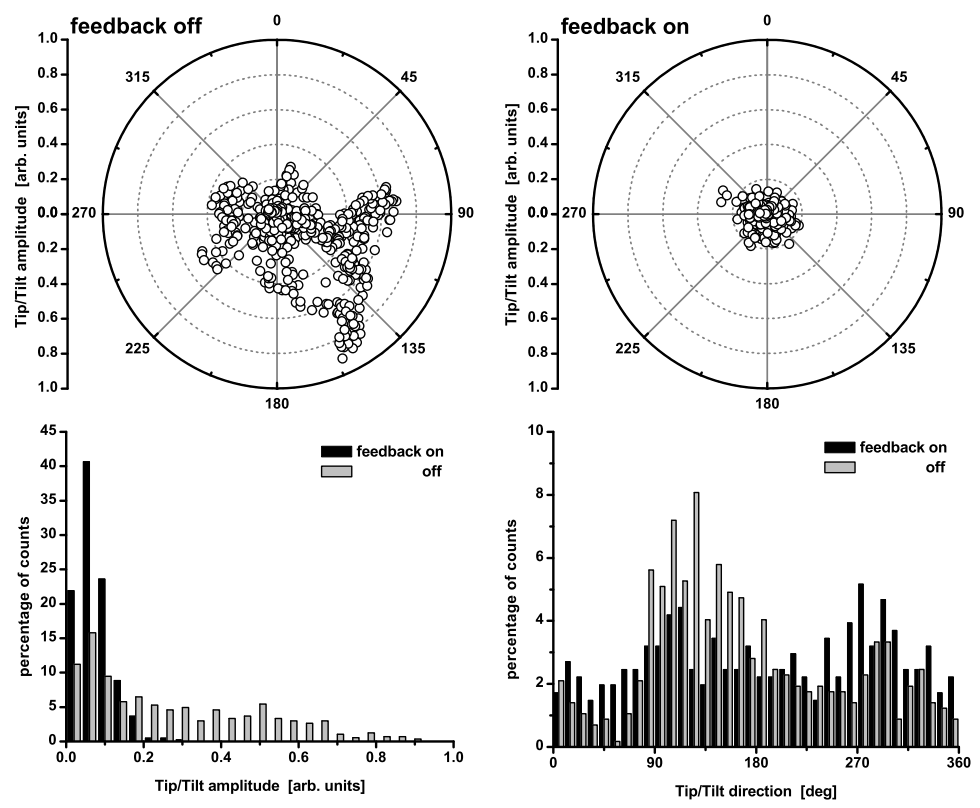

Figure 9. Measurement of the beam deflection without (top left) and with (top right) closing the feedback loop. Analysis of the tip/tilt measurements shows that the liquid mirror system reduces the amplitude (bottom left) and the directional variance (bottom right).
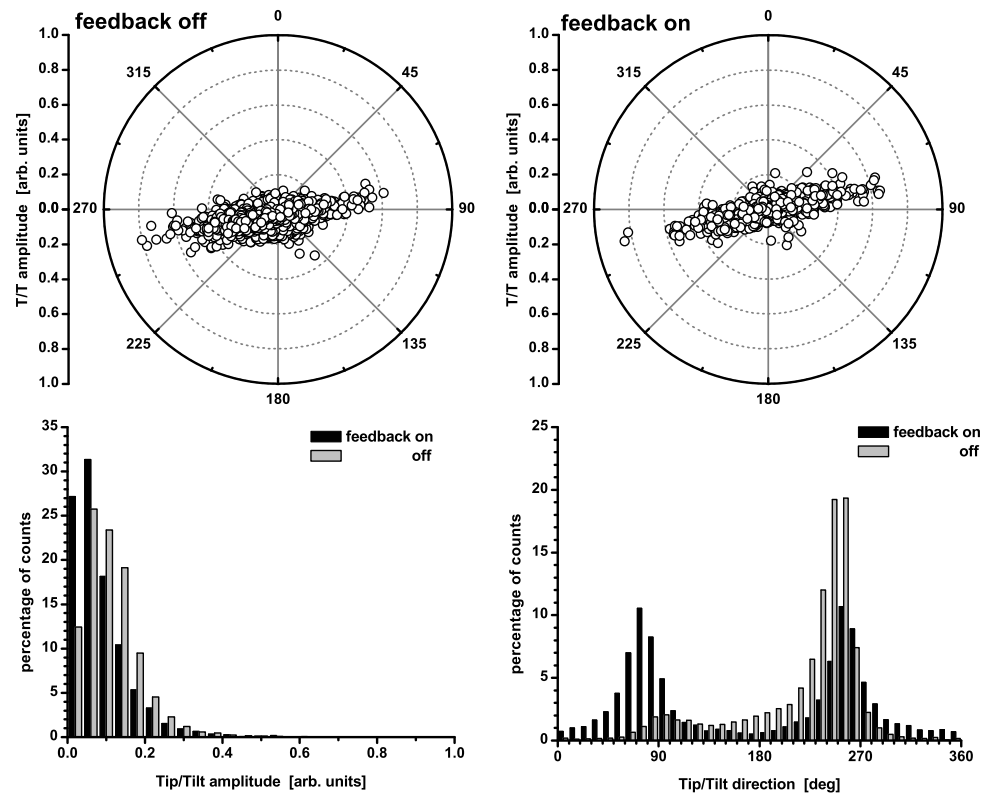

Figure 10. Measurement of the beam deflection without (top left) and with (top right) closing the feedback loop. It can be seen that the vibrations impinging on the liquid mirror system excite the liquid surface along a preferred axis. Analysis of the tip/tilt measurements shows that the liquid mirror system reduces the amplitude (bottom left) and distributes the vibrations symmetrically around the center (bottom right). 


\section{CONCLUSIONS}

The open-loop Bode plot shows that the liquid mirror system is a higher order transfer function with one of the resonance frequencies at about $f_{n}=50 \mathrm{~Hz}$. The gain crossover frequency and the phase crossover frequency of the system are $f_{g c}=1 \mathrm{~Hz}$ and $f_{p c}=250 \mathrm{~Hz}$, respectively. This results in a gain margin of $G M=17 \mathrm{~dB}$ and a phase margin of $P M=179^{\circ}$, predicting that the system is stable.

The closed-loop experiments show that the liquid mirror device is capable of consistently and stably reducing the residual error depending on the specific feedback parameters by a factor of 3 - 4 over a wide range of feedback parameters. A detailed analysis of the tip and tilt modes of a beam aberrated by the phase disk show that the liquid mirror device reduces the amplitude of the deflection by $73 \%$ and the variability of the angular direction by $54 \%$. Observations of the influence of ambient vibrations on the liquid mirror device show a preferred direction of the deflection along the axis oriented along $75^{\circ}-255^{\circ}$. Using the liquid mirror device the amplitude of the vibrations can be reduced by $21 \%$ (Fig. 10, bottom left) and the variability of the angular distribution by $38 \%$. However, here the main effect is the symmetrical distribution of the deflections around the origin (Fig. 10, bottom right).

Fig. 11 shows an analysis of the coefficients of the first ten Zernike modes with and without feedback. Without feedback the strongest modes are $\mathrm{C}[1,1]$ and $\mathrm{C}[1,-1]$ (tip and tilt) for both the measurements with the rotating phase disk (left) and for the ambient vibrations (right). This shows that the main effect of the phase disk is the deflection of the beam from its origin. With the feedback activated a significant reduction of the error can be observed while the size of the remaining error is comparable for all modes. In the case of the ambient vibrations the higher modes are much weaker than tip and tilt, confirming that the ambient vibrations excite large-scale waves on the surface of the liquid that cause a deflection of the beam without causing small scale deformations of the liquid-air interface.
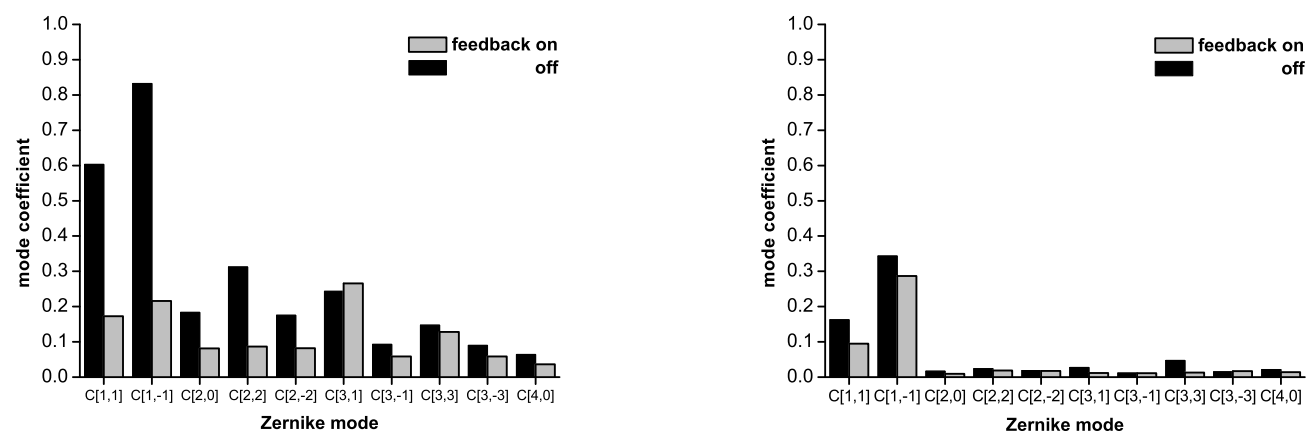

Figure 11. Coefficients of the Zernike modes constituting the error signal without and with closing the feedback loop for the phase disk (left) and ambient vibrations (right).

\section{SUMMARY}

We have measured the open-loop frequency response and the closed-loop feedback performance of a liquid deformable mirror device. The higher-order response predicts a stable operation of the mirror in a feedback system, thus allowing the use of the liquid mirror in a closed-loop AO system. Since the frequency response and feedback characteristics depend on the material properties of the liquid and the geometry of the device used, it may be possible to tune the characteristics of the system in order to optimize the performance of the mirror. However, more work on the influence of the material properties of the liquid has to be done.

In accordance with the predicted stability of the system the liquid mirror performed well in a closed-loop $\mathrm{AO}$ system and did improve a laser beam aberrated by a phase disk. Static and dynamic measurements were performed and showed an average improvement factor in terms of the residual rms-error of $I_{\text {rms,static }}=6.44$ for the static case and of around $I_{\mathrm{rms} \text {,dynamic }}=3.5$ for the dynamic case depending on the system parameters. 
Extended measurements of the tip and tilt modes of the unaberrated beam showed the presence of a preferred direction of oscillation of the beam due to ambient vibrations. It could be shown that by activating the feedback the amplitude could be reduced and the oscillations could be distributed symmetrically around the origin.

\section{ACKNOWLEDGMENTS}

The authors want to thank Dr. Loktev and Dr. Soloviev for many comments, recommendations, and fruitful discussions.

\section{REFERENCES}

[1] Wood, R. W., "The mercury paraboloid as a reflecting telescope," Astrophys. J. 29, 164-176 (1909).

[2] Borra, E. F., Beauchemin, M., and Lalande, R., "Liquid mirror telescopes: Observations with a 1 meter diameter prototype and scaling-up considerations," ApJ 297, 846-851 (October 1985).

[3] Hickson, P., Borra, E. F., Cabanac, R., Content, R., Gibson, B. K., and Walker, G. A. H., "UBC/Laval 2.7 meter liquid mirror telescope," ApJ 436, L201-L204 (December 1994).

[4] Borra, E. F., Ritcey, A. M., Bergamasco, R., Laird, P., Gingras, J., Dallaire, M., Da Silva, L., and YockellLelievre, H., "Nanoengineerd astronomical optics," A\&SA 419, 777-782 (2004).

[5] Vdovin, G., "Closed-loop adaptive optical system with a liquid mirror," Opt. Lett. 34, 524-526 (February 2009). 